\title{
Modeling Material Interfaces with Hybrid Adhesion Method
}

\author{
Nicholas Brown ${ }^{1,2}$, Jianmin $\mathrm{Qu}^{1}$, Enrique Martinez ${ }^{2}$ \\ ${ }^{1}$ Northwestern University; 2145 Sheridan Road, Room B224, Evanston, IL 60208 \\ ${ }^{2}$ Material Science and Technology Division; MST-8, Los Alamos National Laboratory, Los \\ Alamos, 87545 NM, USA
}

\begin{abstract}
A molecular dynamics simulation approach is presented to approximate layered material structures using discrete interatomic potentials through classical mechanics and the underlying principles of quantum mechanics. This method isolates the energetic contributions of the system into two pure material layers and an interfacial region used to simulate the adhesive properties of the diffused interface. The strength relationship of the adhesion contribution is calculated through small-scale separation calculations and applied to the molecular surfaces through an inter-layer bond criterion. By segregating the contributions into three regions and accounting for the interfacial excess energies through the adhesive surface bonds, it is possible to model each material with an independent potential while maintaining an acceptable level of accuracy in the calculation of mechanical properties. This method is intended for the atomistic study of the delamination mechanics, typically observed in thin-film applications. Therefore, the work presented in this paper focuses on mechanical tensile behaviors, with observations in the elastic modulus and the delamination failure mode. To introduce the hybrid adhesion method, we apply the approach to an ideal bulk copper sample, where an interface is created by disassociating the force potential in the middle of the structure. Various mechanical behaviors are compared to a standard EAM control model to demonstrate the adequacy of this approach in a simple setting. In addition, we demonstrate the robustness of this approach by applying it on (1) a $\mathrm{Cu}-\mathrm{Cu}_{2} \mathrm{O}$ interface with interactions between two atom types, and (2) an $\mathrm{Al}-\mathrm{Cu}$ interface with two dissimilar FCC lattices. These additional examples are verified against EAM and COMB control models to demonstrate the accurate simulation of failure through delamination, and the formation and propagation of dislocations under loads. The results conclude that by modeling the energy contributions of an interface using hybrid adhesion bonds, we can provide an accurate approximation method for studies of large-scale mechanical properties, as well as the representation of various delamination phenomena at the atomic scale.
\end{abstract}

Keywords: Atomic Interface, Adhesion Energy, Molecular Dynamics, Inter-Atomic Potential, Interfacial Free Energy

\section{Introduction}

A common complication associated with MD simulations is the definition and selection of interatomic force fields. These force fields provide parameters used to calculate the atom-toatom interaction behaviors for the simulations. While there are numerous types of force fields available for isolated systems[1], there remains a limited amount of intermolecular capabilities 
for complex systems. Organizations, such as the National Institute of Standards and Technology (NIST) and the Knowledge of Interatomic Models (KIM), provide researchers with a vast data repository of potentials for use in both MD and ad-initio simulations. However, due to the complex nature of these potentials, the applications of each force field are often limited in scope and can provide unrealistic results if misused. One example is the Embedded Atom Method (EAM) force field, which is a well-defined approach used to simulate structural, mechanical and thermal behaviors of some pure metal structures [2] [3]. While this force field is well defined and verified for materials such as pure copper and aluminum, it requires additional adjustments when observing additional atom interactions, such as the effects of surface oxidation [4]. Another approach that is being continuously developed is the Charge-Optimized Many Body (COMB) potential for various metal-oxides. The use of these potentials are typically restricted to temperature-dependent oxidation growth [5] [6], low mechanically strained systems or lowtemperature interfaces [7]. The issue grows when the scope of the simulation includes additional complex interactions, such as those between metals and polymers. The potentials used to model polymers (PCFF, AMBER, CHARMM, etc.) are fundamentally different and incompatible to those used for metallic structures (EAM, MEAM or COMB), this creates a computational challenge when modeling the composite interfaces. With no direct relationship between these force fields, often a new force field relationship must be developed on a material specific basis [8]. As an alternative method, we propose a hybrid adhesion method (HAM) to approximate the mechanical characteristics of surface interactions by linking two or more discrete force fields by an array of adhesive energy bonds.

The principle concept behind the hybrid adhesion method is the simplification of the complex behaviors at a material interface. The theory was developed by combining the principles of classical mechanics used in MD simulations, with those of density functional theory (DFT) in quantum mechanics. The result is a hybrid potential model that enables the use of multiple independent force fields as well as reduces the total computational time by limiting the material interactions to a surface plane at the interface. The method is described in this paper and demonstrated on three different interfaces for application and verification purposes. The first interface is of a standard bulk copper structure modeled with an EAM potential, where the structure is split into layers and the interatomic potentials are discretized to act as independent entities. The second interface is between copper and cuprous-oxide, modeled using the COMB potential. Like the bulk copper structure, the $\mathrm{Cu}-\mathrm{Cu}_{2} \mathrm{O}$ model is separated into layers of independent force fields and provides a complicated interface structure with multiple atomic interactions. The oxidation of copper surfaces is often observed in electrical components and 3D packaging where it is stacked with thin layers of an epoxy compound [9] [10] and provides a good introductory interface for future studies. The final structure is a dissimilar face-centered cubic (FCC) interface of aluminum and copper using an EAM potential. The model introduces the hybrid adhesion method to a misfit dislocation interface. Multi-layered stacking is prone to delamination through fluctuations in environmental conditions, making it an ideal case study for this modeling technique. As such, the application and verification of this method is focused on the mechanical properties and behaviors of each material interface as well as an in-depth study of delamination. To verify the hybrid adhesion method, controlled models using traditional MD techniques were used as baseline structures for comparisons mechanical behavior and response. 


\section{Theoretical Background}

The theory behind the hybrid adhesion method originates from the concepts of energy distribution presented by Gibbs and the ideal interface model, Figure 1. Energy contributions of a two-phase structure consist of a bulk concentration of $\alpha$-phase, a bulk concentration of $\beta$-phase and a surface phase marked by the diffused region at the interface. The energy contribution associated with this surface phase is known as interfacial free energy, or interfacial excess energy[11-13]. A dividing surface can be visualized through a change in energy density profile, normal to the interface, Figure 1(b). The profile displays two constant energy densities for the bulk phases, and a deviating function associated with the contributions of the surface phase region.

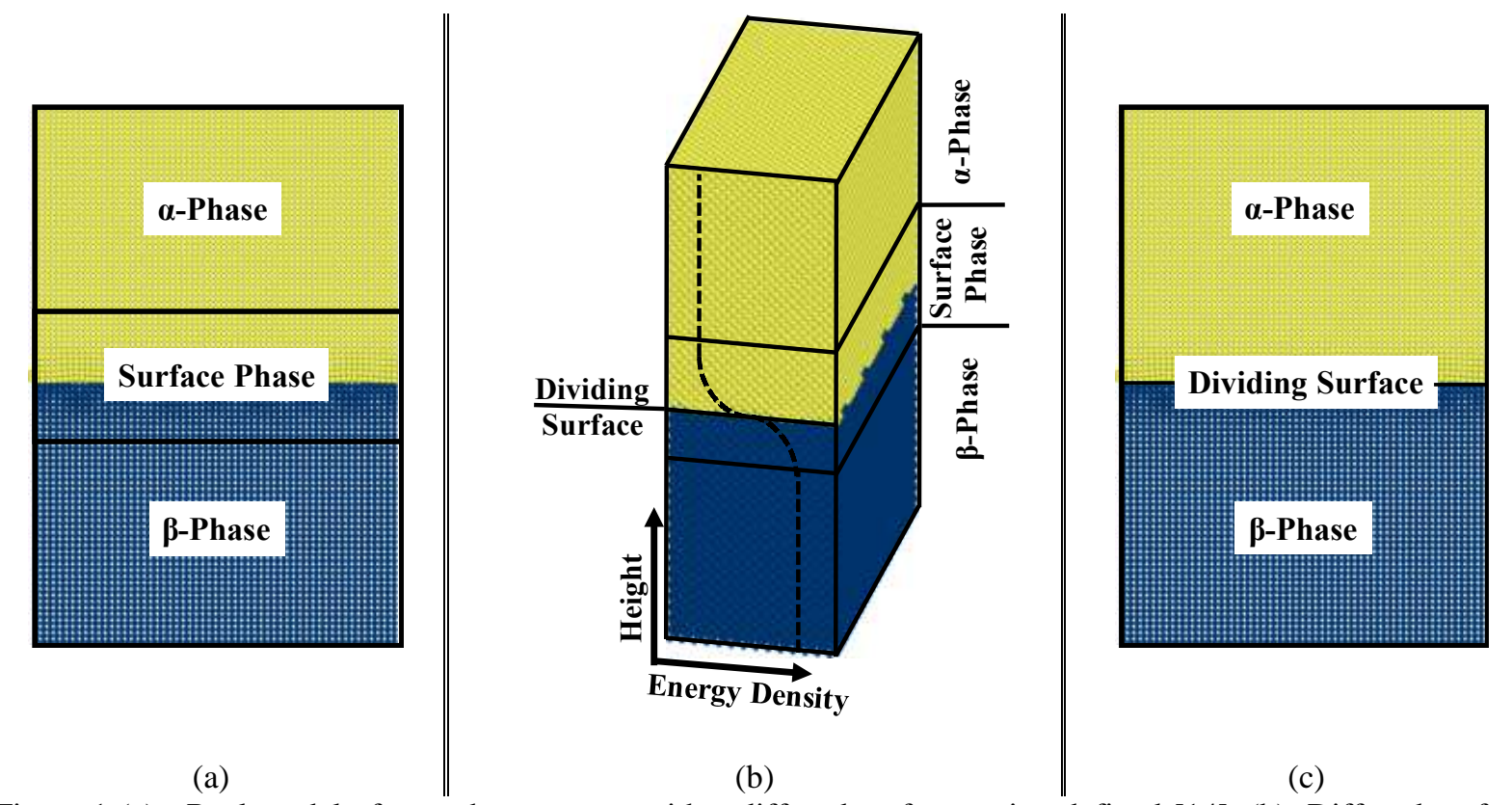

Figure 1 (a): Real model of two-phase system with a diffused surface region defined [14]. (b): Diffused surface region defined by energy densities [15]. (c): Gibbs Ideal Interface with infinitely thin dividing surface [14].

With the surface phase layer proportionally thin in comparison with other dimensions[13], Gibbs introduced the concept of a dividing surface where the surface phase is idealized as an interface separating two homogeneous phases. By reducing the interface to an infinitely thin plane, the total energy of the system can be reduced to the sum of the atomic contributions in the two phases in addition to an interfacial free energy, $\gamma$. For an ideal two-phase system (Figure $1(\mathrm{c}))$, the corresponding interfacial excess energy can be approximated by:

$$
\gamma(T)=\frac{E_{t o t}-\left(N_{\alpha} e_{\alpha}+N_{\beta} e_{\beta}\right)}{A}
$$

where $A$ is the surface area of the interface, $N$ is the number of atoms in the respected phases and $e$ is the potential energy per atom in the bulk state[16]. The top term, $E_{\text {tot }}-\left(N_{\alpha} e_{\alpha}+N_{\beta} e_{\beta}\right)$, is also referred to boundary enthalpy and is often used in MD studies of grain boundaries and applications of mobility[16-19]. The development of the hybrid adhesion method presented in this paper applies the same principles and approximations to apply an adhesive bonding to two, otherwise independent, materials. 


\subsection{Hybrid Adhesion Method}

The hybrid adhesion method was developed following similar concepts as Gibbs Ideal Interface model, where it discretizes the structure into three partitions: bulk $\alpha$-material, bulk $\beta$ material and an adhesive region. If two bulk materials are defined as independent in both energy and force interactions, the interfacial excess energy and adhesion characteristics can be limited to a controlled adhesive region. Such that, under equilibrium conditions, the adhesion potential contributes the same amount of energy as the previously identified Gibbs dividing surface. As the two surfaces begin to separate, the additional contributions are determined by a relationship defined using an adhesion function. Similar relationships have been used to define adhesionforce characteristics of complex interfaces, such as metal-oxides and epoxy[20]. Where small scale first-principle density functional theory (DFT) calculations are used to calculate the adhesion separation relationship across a surface area. Unlike the classical mechanics of molecular dynamics (MD) simulations, DFT is not limited by the availability of interatomic potentials and offers the freedom to study unlimited combinations of atom interactions. However, since the model size is the biggest restriction in DFT calculations, this approach is limited to small-scale models and requires multi-scale simulations to study misfit dislocations and defects. The hybrid adhesion method provides a means of multi-scale simulations by expanding on these adhesion force calculations and applying the resulting relationships to MD applications. The models possess the capabilities of simulating larger-scale interfaces for applications in mechanical loading. In addition, more recent studies in DFT have included the effects of surface moisture [21,22] on adhesive characteristics, which paves the path for future inclusions on large-scale simulations through the application of the hybrid adhesion method.

To apply the first principle adhesive characteristics to a MD simulation, it is first necessary to identify the independent adhesive region. This region that can be thought of as a network of inelastic springs that join the two surfaces together, Figure 2, where the total energy associated with the hypothetical springs is equal to the adhesion energy per unit area calculated through DFT. In MD simulations, artificial bonds can be added between the first layer of atoms on each surface to create an adhered surface relationship capable of transferring energy and forces between layers. The strength of the added bonds is calibrated with the small-scale calculations using the total number of bonds added and the bond lengths as a function of separation distance. In a face-centered cubic (FCC) lattice case, such as $\mathrm{Cu}-\mathrm{Cu}$, Figure 3 (a.), the separation distance can be related to the bond length through the initial geometric properties of the lattice. However, in more complex realistic interfaces where there are multiple atom type interactions or misfit dislocations, Figure 3 (b.) and (c.), the equilibrium bond distance will vary based on each individual bond requiring additional calibrations in the energy separation calculation. 


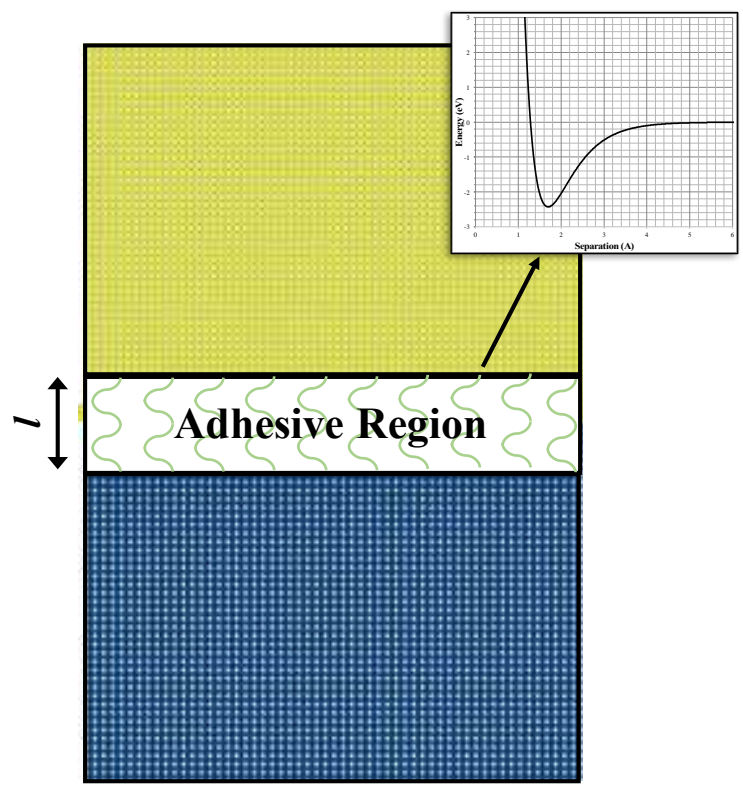

Figure 2: Simulated hybrid adhesion method with interface characteristics approximated using adhesion bond region. (a.)

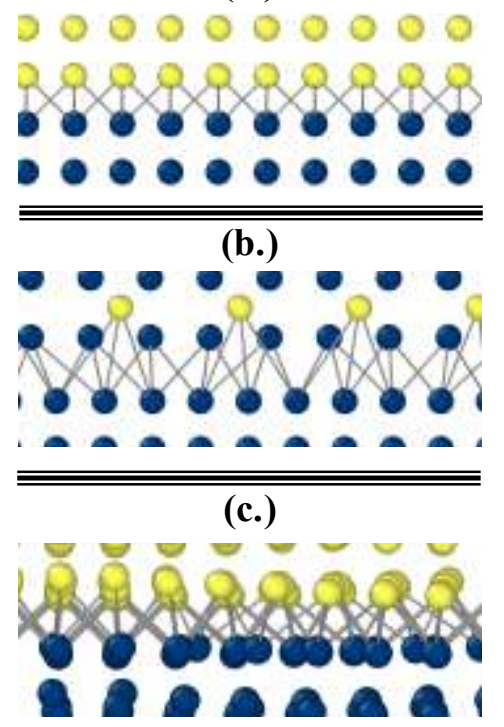

Figure 3: (a.) $\mathrm{Cu}-\mathrm{Cu}$ (100) interface with adhesion bonds (b.) $\mathrm{Cu}-\mathrm{Cu} 2 \mathrm{O}$ (100) interface with adhesion bonds (c.) $\mathrm{Al}-\mathrm{Cu}(100)$ interface with adhesion bonds

We now return to the concept of ideal interface and incorporate the separation distance, $l$, resulting in an interfacial excess adhesive energy per surface area, $\gamma_{a d}$, where $E_{\text {tot }}$ is a function of separation distance and $N_{\alpha} e_{\alpha}+N_{\beta} e_{\beta}$ can be calculated as the total energy at an infinitely large separation distance:

$$
\gamma_{a d}(T, l)=\frac{E_{t o t}-\left(N_{\alpha} e_{\alpha}+N_{\beta} e_{\beta}\right)}{A}
$$

If we identify the number of bonds used to define the adhesion region as $N_{b}$, and the energy contribution of each bond as $\gamma_{m=1 \ldots N_{b}}$, we can define the total potential energy of the system as the sum of three independent interatomic potential contributions:

$$
\begin{gathered}
E_{\text {tot }}=E_{\alpha}+E_{\beta}+E_{a d} \\
E_{t o t}=\sum_{i, j=1}^{N_{\alpha}} u^{\alpha}\left(r_{i j}{ }^{\alpha}\right)+\sum_{k, l=1}^{N_{\beta}} u^{\beta}\left(r_{k l}{ }^{\beta}\right)+\sum_{m=1}^{N_{b}} \gamma_{m}\left(r_{b}\right)
\end{gathered}
$$

where $r$ is defined as the distance between atoms, or the bond length for the adhesion contribution and $E_{a d}=A \gamma_{a d}=\sum_{m=1}^{N_{b}} \gamma_{m}\left(r_{b}\right)$. The benefit of the hybrid adhesion method is that the potential contributions are independent, which allows each material layer to be modeled with a different force potential, $u$. This can be especially useful when modeling the delamination of complex composite interfaces of metals, metal-oxides and polymers[8, 10], often seen in consumer electronic studies, where a combination of potentials can be used in a single simulation. The following section highlights the benefits and applicability of this method through the detailed walkthrough using a simple $\mathrm{Cu}-\mathrm{Cu}(001)$ and more complex $\mathrm{Al}-\mathrm{Cu}(001)$ and $\mathrm{Cu}-$ $\mathrm{Cu}_{2} \mathrm{O}$ (001) interfaces under load conditions. 


\section{Method Application}

The hybrid adhesion method can be applied using LAMMPS Molecular Dynamics Simulator [23]. This section explains in detail the $\mathrm{Cu}-\mathrm{Cu}(001)$ interface modeled with the EAM potential: (1) development of adhesion-separation relationship, (2) development of adhesion bonds and the (3) application and calibration of adhesion bonds. The following, Section 4, will provide additional verification results for $\mathrm{Al}-\mathrm{Cu}$ and $\mathrm{Cu}-\mathrm{Cu}_{2} \mathrm{O}$ interfaces as well as results from multiple interatomic potential combinations.

\subsection{Development of Adhesion-Separation Relationship}

The strength of the adhesion bonds can be calculated through small-scale first principle DFT calculations [20, 22, 24-26], however, in this study, the adhesion relationship is approximated using LAMMPS MD simulations to reduce computation time and limit the additional outside influences for verification purposes. The simulation box for the MD calculations of adhesion energy is configured similarly to DFT methods, with periodic boundary conditions containing two layers of $\mathrm{Cu}(001)$ and a large vacuum space in the direction of separation, Figure 4 . The vacuum is used to negate the effects of the periodic boundary while separating the structure. Each layer of copper is defined by a supercell structure of $8 \times 8 \times 8$ repeating lattices. The two structures are stacked in the $\mathrm{z}$-direction resulting in a combined size of 4,224 atoms.

The copper atoms are modeled using an EAM/alloy potential[27] and the energy of the structure is initially minimized using a zero external pressure constraint. Prior to calculating the energy profile, the top layer of $\mathrm{Cu}$ is displaced closer to the surface of the bottom layer to include the repulsive forces of the interaction. The system energy, at varying distances of separation, is used to approximate the tensile delamination failure mode. From Equation ( 2 ), the equilibrium distance is identified at the minimum energy distance and $N_{\alpha} e_{\alpha}+N_{\beta} e_{\beta}$ is identified where energy becomes constant, Figure 5.

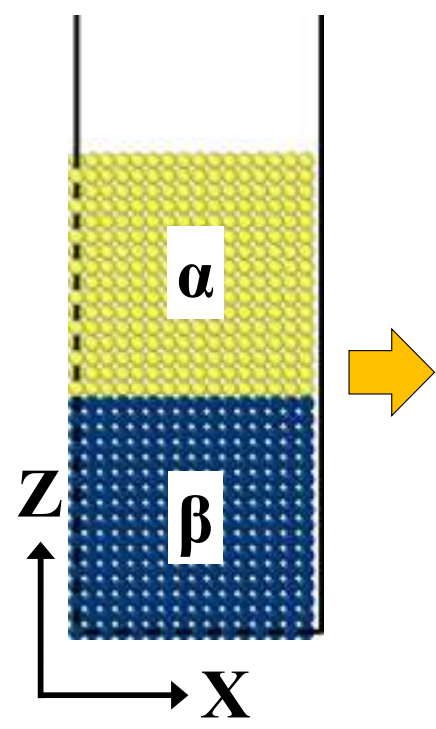

Figure 4: Small-scale energy-separation simulation box used to calculated adhesion relationship in MD simulation

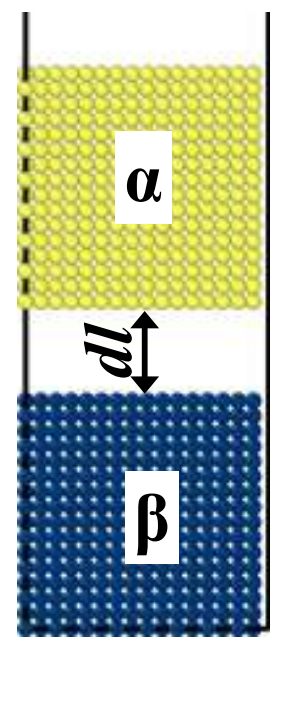

Figure 6 highlights some of the steps taken to convert the total energy to the final adhesion bond energy using the mean bond lengths as a function of separation distance. The adhesion 
bond energy, $\gamma_{m}$, is a function of the number of bonds defined at the interface using the ideal geometry and based on a bond selection criteria detailed in Section 3.3.

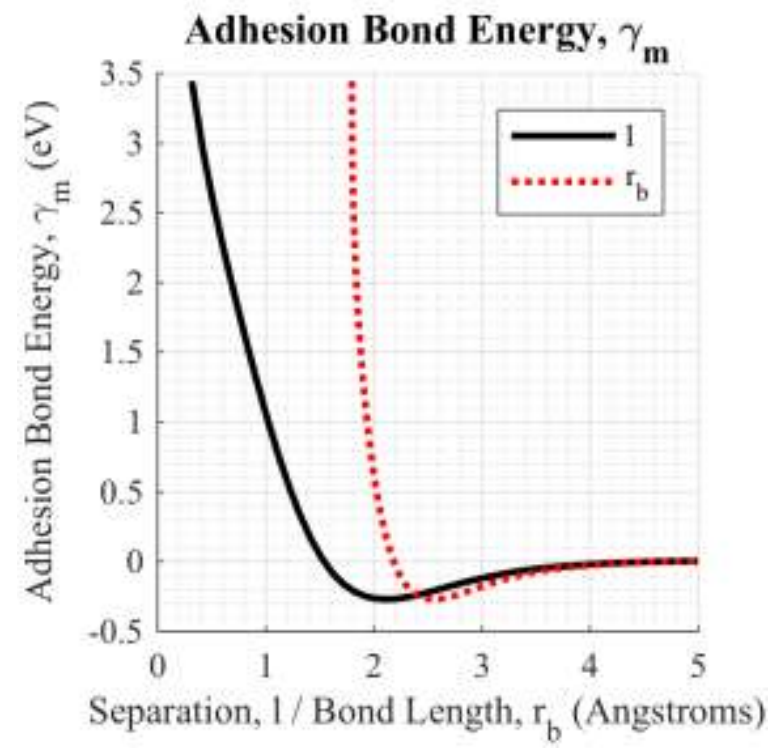

Figure 6: Excess adhesion energy, $\gamma_{a d}$, of $\mathrm{Cu}-\mathrm{Cu}$ (001) interface as a function of surface separation and adhesion bond lengths.

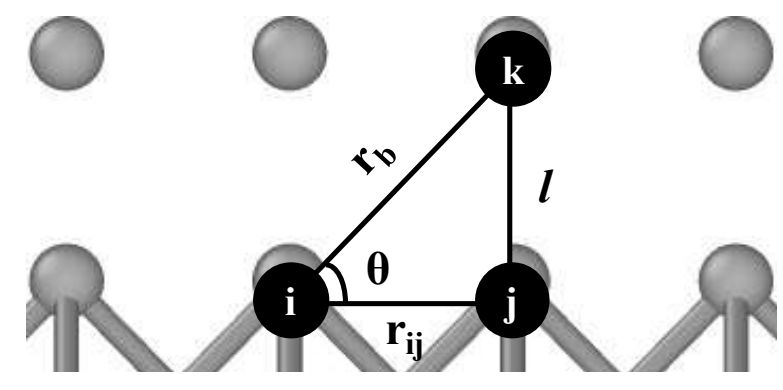

Figure 7: Bond criteria used to link $\mathrm{Cu}-\mathrm{Cu}(001)$ interface with adhesion bonds and calculated bond length as a function of separation distance.

\subsection{Development of Adhesion Bonds}

To apply the adhesion-separation relationship to the hybrid adhesion method, we must first define a bond criterion to create links between the two independent surfaces. For metallic structures, such as the $\mathrm{Cu}-\mathrm{Cu}$ interface, the ideal bonding criterion uses a cutoff distance to link the surface atoms of one layer to the other, Figure 7. For the $\mathrm{Cu}-\mathrm{Cu}$ case, the cutoff distance was set to $3.615 \AA$ resulting in a total of 512 bonds. The geometry of a face-centered cubic lattice, such as copper, results in bonds lengths, $r_{b}$, angled at the known angle of $\theta=45^{\circ}$. For this ideal case where $r_{i j}$ is constant during separation, the bond length can be linked to the separation distance, $l$, through the trigonometric function; $r_{b}=\frac{l}{\cos \theta}$. Alternatively, the equivalent mean bond length, as a function of separation distance, can be numerically extracted from the adhesion separation calculations, Figure 8. Note: If the interface is defined by multiple length adhesion bonds, and the mean bond length is used, the bond strength will need to be calibrated to match the energy-separation relationship. The hybrid adhesion method is ideal for the atomically flat interfaces of metallic structures including cleaved coarse-grain models where multiple bond lengths exist. 


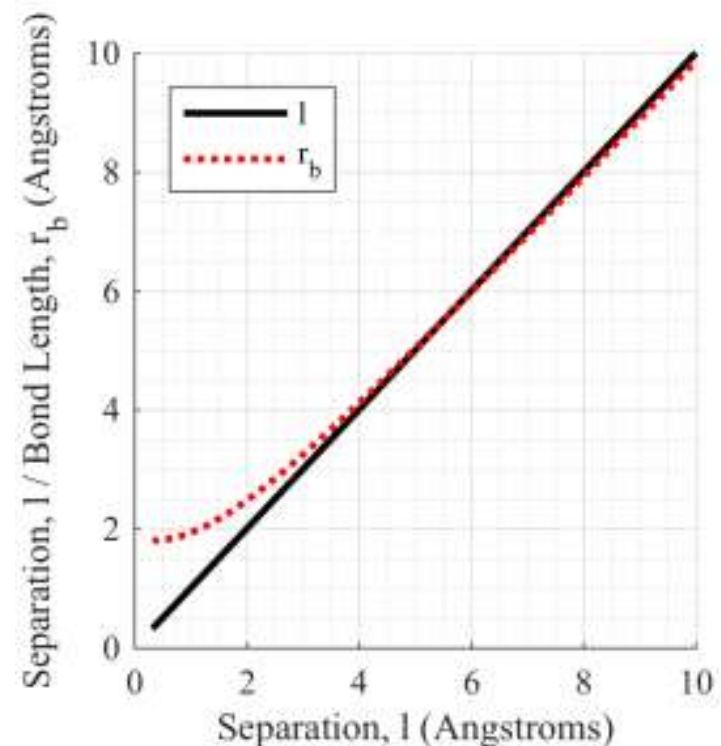

Figure 8: Separation distance to bond length approximation for $\mathrm{Cu}-\mathrm{Cu}(001)$ interface.

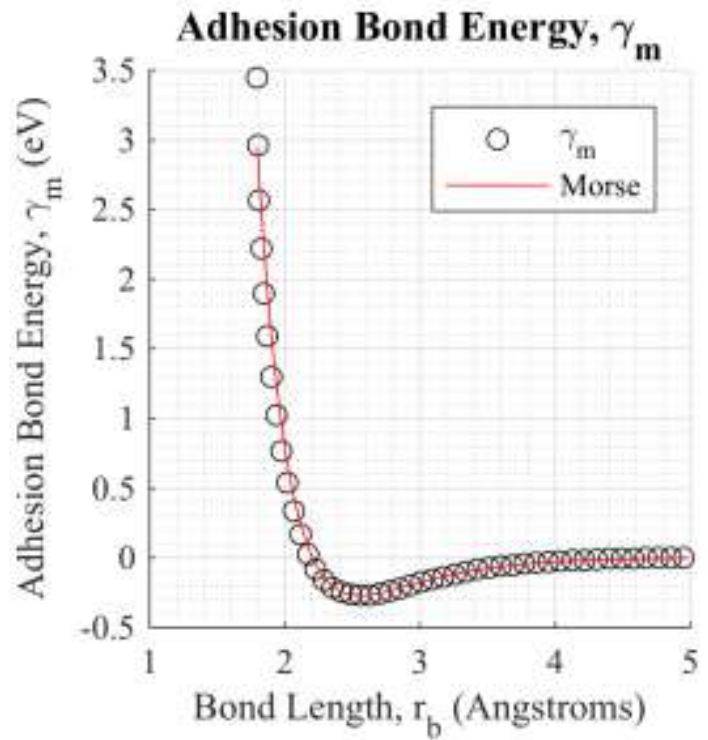

Figure 9: Adhesive energy contributions per bond, $\gamma_{m}$, for $\mathrm{Cu}-\mathrm{Cu}$ (001) interface.

By numerically approximating the initial calculation of bond strength with a mathematical function, we can interpolate and extrapolate the relationship and apply it to MD simulations. This approximation is case-dependent and will vary based on the material interface interactions. The relationship of the $\mathrm{Cu}-\mathrm{Cu}$ interface example emulates a Morse potential[28] defined as:

$$
U(r)=D\left[e^{-2 a\left(r-r_{e}\right)}-2 e^{-a\left(r-r_{e}\right)}\right]
$$

where $D$ is a constant related to the depth of the energy well, $a$ is a shape parameter of the curve and $r_{e}$ is equal to the equilibrium distance. In this method, the equilibrium distance is equivalent to the mean bond length at the minimum energy state. For the copper interface sample with 512 defined adhesion bonds, the adhesive energy carried per bond is calculated by multiplying the bond length factored excess adhesion energy by the interface surface area and divided by the number of bonds, Equation ( 4 ). The resulting energy per bond, $\gamma_{m}$, and its Morse approximation are shown in Figure 9. For most interfaces, the bond lengths will be a statistical distribution of length and will require additional calibration of the functions constant parameters, or $a$ and $D$ in the case of the Morse approximation. More details on the necessary verification methods are provided in the following Section 3.3.

\subsection{Application and Calibration of Adhesion Bonds}

The goal of the hybrid adhesion method is to provide an approximate relationship of the adhesive qualities of a multi-layered interface, and requires additional calibration of bond strength parameters. With the known adhesive energy contribution per bond, we can apply the 512 bonds to the adhesion-separation model, described in Section 3.1 and shown in Figure 4, using the defined bond criteria, maximum cutoff distance of $3.615 \AA$. We disassociate the EAM interatomic potential of the top layer from the bottom layer to ensure the only reactions are those controlled by the adhesion bonds. With the structure now discretized into three regions and the interfacial characteristics applied only through the adhesion bonds, we can repeat a variation of the energy-separation simulation from Section 3.1. The energy-separation relationship can then be compared to the control model, Figure 10. 


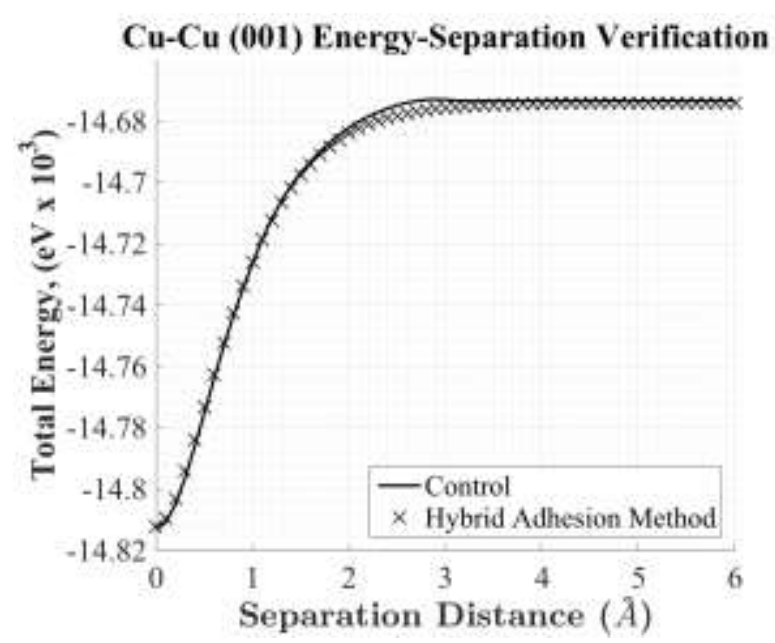

Figure 10: Verification of energy-separation relationship for $\mathrm{Cu}-\mathrm{Cu}$ (001) interface, hybrid adhesion method compared to control, EAM, model. Separation distance is defined as the distance away from the equilibrium position.

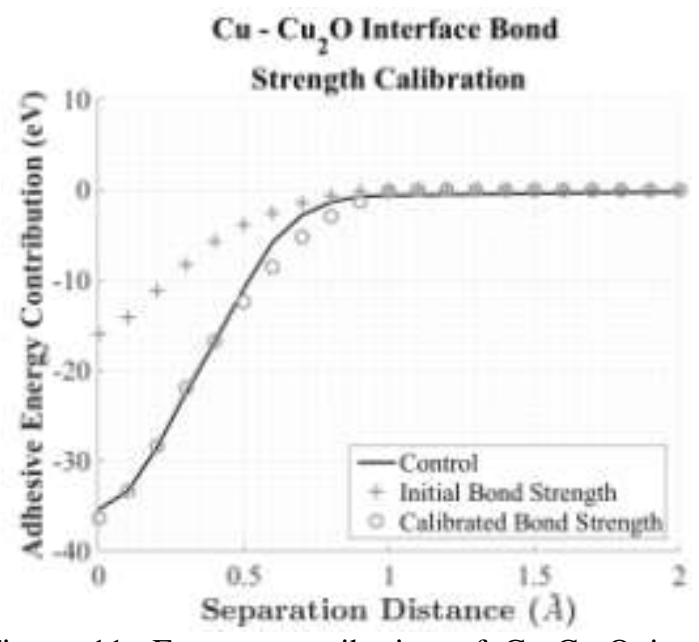

Figure 11: Energy contribution of $\mathrm{Cu}-\mathrm{Cu}_{2} \mathrm{O}$ interface due to adhesion characteristics. Control model is with the COMB potential. The calibrated bond strength denotes the hybrid adhesion method results.

The purpose of the hybrid adhesion method is to provide an approximate relationship of the adhesive qualities of a multi-layered interface. Although the sample $\mathrm{Cu}-\mathrm{Cu}$ interface results in an accurate representation of the adhesion-separation relationship, more complex interfaces require additional calibrations of bond strength parameters. For example, the interface between $\mathrm{Cu}-\mathrm{Cu}_{2} \mathrm{O}$ (001), where non-uniform bonds are added between the $\mathrm{Cu}-\mathrm{Cu}$ surface interface and the $\mathrm{Cu}-\mathrm{O}$ surface interface. In such cases, the adhesion energy well depth and width parameters of the bond strength must be modified to calibrate for the energy-separation relationship, Figure 11. With the energy-separation relationship of the hybrid adhesion method fit to the controlled case, we can apply mechanical loading conditions and observe the dynamic behaviors.

\section{Results}

This section provides results of three material interfaces modeled using the hybrid adhesion method described in Section 3. For verification, control models were initially developed to provide baseline simulation results using single interatomic potentials: (a.) Full copper structure modeled with the EAM potential consisting of three $\mathrm{Cu}$ layers (b.) $\mathrm{Cu}-\mathrm{Cu}_{2} \mathrm{O}-\mathrm{Cu}$ sandwich structure modeled using a COMB potential (c.) Large scale two-layer structure consisting of $\mathrm{Al}$ and $\mathrm{Cu}$ EAM materials, used primarily to demonstrate the delamination failure mode, Figure 12. A summary of the adhesion bond parameters used for these three models are provided in Table I and Figure 13. 


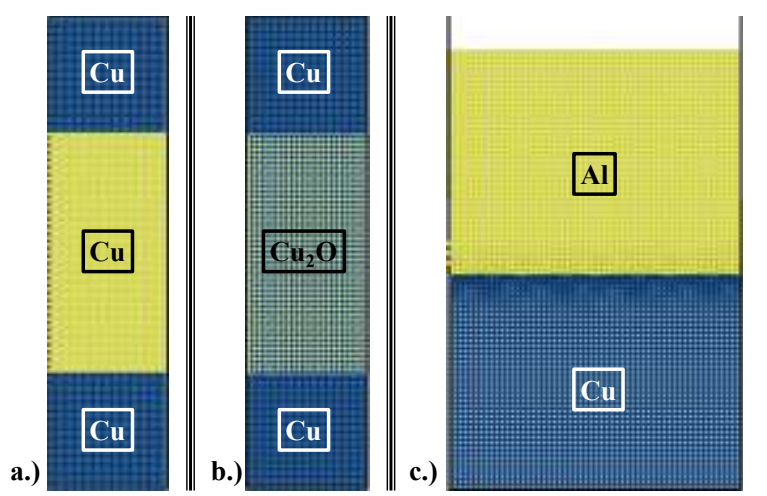

Table I: Adhesion bond properties

\begin{tabular}{|c|c|c|c|c|c|c|c|}
\hline & $\alpha$ & B & Bond & $r_{e}$ & $D$ & $a$ & $r_{c}^{\text {III. }}$ \\
\hline a. & $\mathrm{Cu}$ & $\mathrm{Cu}$ & Morse $^{I}$ & 2.559 & 0.273 & 1.963 & 3.615 \\
\hline b. & $\mathrm{Cu}$ & $\mathrm{Cu} 2 \mathrm{O}$ & Harmonic $^{\text {II }}$ & 3.102 & 0.361 & 0.530 & 4.267 \\
\hline c. & $\mathrm{Al}$ & $\mathrm{Cu}$ & Morse $^{\mathrm{I}}$ & 2.650 & 0.272 & 1.650 & 4.0495 \\
\hline
\end{tabular}

Figure 12: (a) Bulk MD model of $\mathrm{Cu}-\mathrm{Cu}-\mathrm{Cu}$, full periodic boundary conditions. (b) $\mathrm{Cu}-\mathrm{Cu}_{2} \mathrm{O}-\mathrm{Cu}$ sandwich structure, periodic boundary conditions. (c) $\mathrm{Al}-\mathrm{Cu}$ interface with free surface in z-direction.

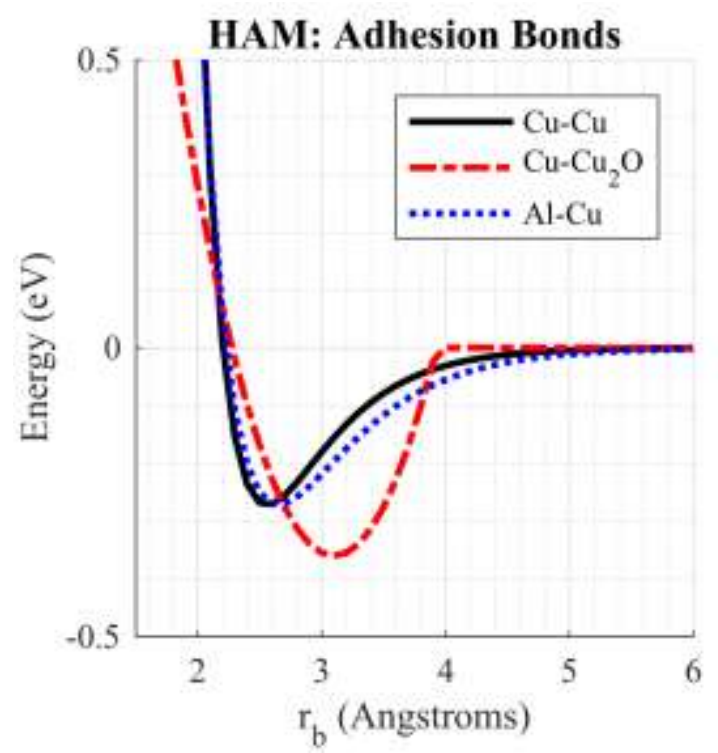

Figure 13: Adhesion bond strengths for $\mathrm{Cu}-\mathrm{Cu}, \mathrm{Cu}-$ $\mathrm{Cu}_{2} \mathrm{O}$ and $\mathrm{Al}-\mathrm{Cu}$ interfaces.

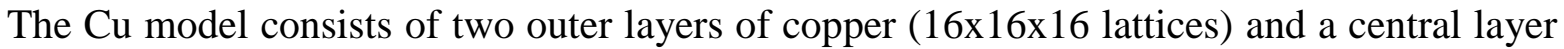
of copper (16x16x32 lattices) for a total of 65,536 atoms. The $\mathrm{Cu}_{2} \mathrm{O}$ model is built using two outer layers of copper $(15 \times 15 \times 15$ lattices) and a central layer of cuprous-oxide $(12 \times 12 \times 24$ lattices) for a total of 33,192 atoms. The interfaces have been cleaved to create a copper to copper surface between $\mathrm{Cu}$ and $\mathrm{Cu}_{2} \mathrm{O}$. The delamination model an $\mathrm{Al}-\mathrm{Cu}$ interface uses a lattice structure of $14 \times 14 \times 21$ for aluminum and 16x16×24 for copper, resulting in a total of 164,160 atoms. Equivalent structures were developed using the hybrid adhesion method and isolating the force potentials of different material layers in each model. There are 4,096 adhesion bonds added to the copper model, 4,156 bonds added to the cuprous model and 9,488 bonds added to the Al$\mathrm{Cu}$ model. The copper and copper-oxide structures are assigned periodic boundary conditions and initially minimized and equilibrated for 10,000 steps using an NPT ensemble with zero external pressure, $0.1 \mathrm{~K}$ temperature and a time step of $1 \mathrm{fs}$. The $\mathrm{Al}-\mathrm{Cu}$ structure is assigned periodic boundaries in the $\mathrm{x}$ and $\mathrm{y}$ directions and a free surface defined the in the $\mathrm{z}$ to allow for full delamination. 
As an initial verification of the hybrid adhesion method, we calculate the mechanical material properties of the structurally optimized bulk copper model. The calculated properties using the hybrid adhesion method are in good agreement with the EAM MD simulations and experimental data for a bulk substance, Table II:

Table II: Material properties extracted from MD simulations. Hybrid adhesion method (HAM) and embedded-atom method (EAM) results presented in this paper.

\begin{tabular}{|c|c|c|c|c|c|c|c|}
\hline & & $\mathrm{C}_{11}(\mathrm{GPa})$ & $\mathrm{C}_{12}(\mathrm{GPa})$ & $\mathrm{C}_{44}(\mathrm{GPa})$ & $\mathrm{B}(\mathrm{GPa})$ & $\mathrm{G}(\mathrm{GPa})$ & $v$ \\
\hline \multirow{3}{*}{ Bulk Copper } & EAM & 169.9 & 122.6 & 76.2 & 138.4 & 76.2 & 0.419 \\
\cline { 2 - 8 } & $\mathrm{HAM}$ & 170.9 & 124.4 & 77.1 & 139.5 & 77.3 & 0.418 \\
\cline { 2 - 8 } & Exp.[29] & 170 & 122.5 & 75.8 & - & - & - \\
\hline
\end{tabular}

To demonstrate the validity of the hybrid adhesion method in studies of large-scale mechanical properties, the $\mathrm{Cu}-\mathrm{Cu}-\mathrm{Cu}$ structure is initially subjected to uniaxial tensile loading following an equilibration step. The full bulk periodic system is assigned a NPT ensemble with temperatures of $0.1 \mathrm{~K}$ and $300 \mathrm{~K}$, and external pressure constrained to zero in the $\mathrm{x}$ and $\mathrm{y}$ axis. The temperature and pressure of the system are controlled using Langevin and Berendsen fixes respectively. The simulation box was deformed in the z-direction at two constant engineering strain rates of $10^{10} \mathrm{~s}^{-1}$ and $10^{11} \mathrm{~s}^{-1}$. While these strain rates are high compared to experimental data, they do represent typical rates for MD simulations where the material scale is limited to the nano-scale where the statistical effect of material defects does not affect the material strength. As such, the yield and ultimate strengths are often observed to be much higher than the available experimental data. However, the purpose of these simulations is to display the capabilities of the hybrid adhesion method in representing a traditional force field interface under mechanical loading. The hybrid adhesion method shows a good approximation of the stress-strain relationship when compared to the full EAM control model at $0.1 \mathrm{~K}$ and $300 \mathrm{~K}$ and was repeated for the $\mathrm{Cu}-\mathrm{Cu}_{2} \mathrm{O}-\mathrm{Cu}$ structure, Figure 14 through Figure 17.

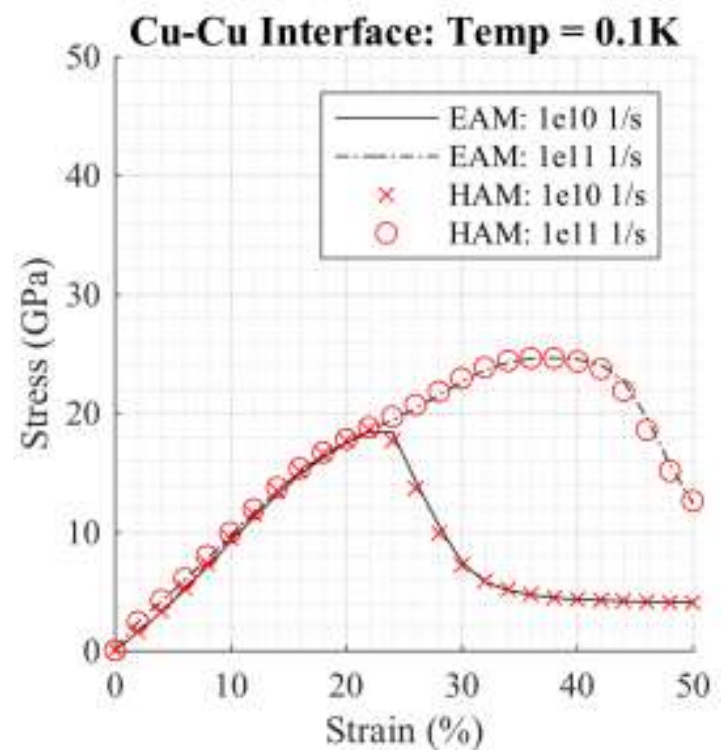

Figure 14: Stress-strain relationship of $\mathrm{Cu}$ control model, EAM, compared to hybrid adhesion method (HAM) at $0.1 \mathrm{~K}$ temperature and strain rates $10^{10}$ and $10^{11} 1 / \mathrm{s}$.

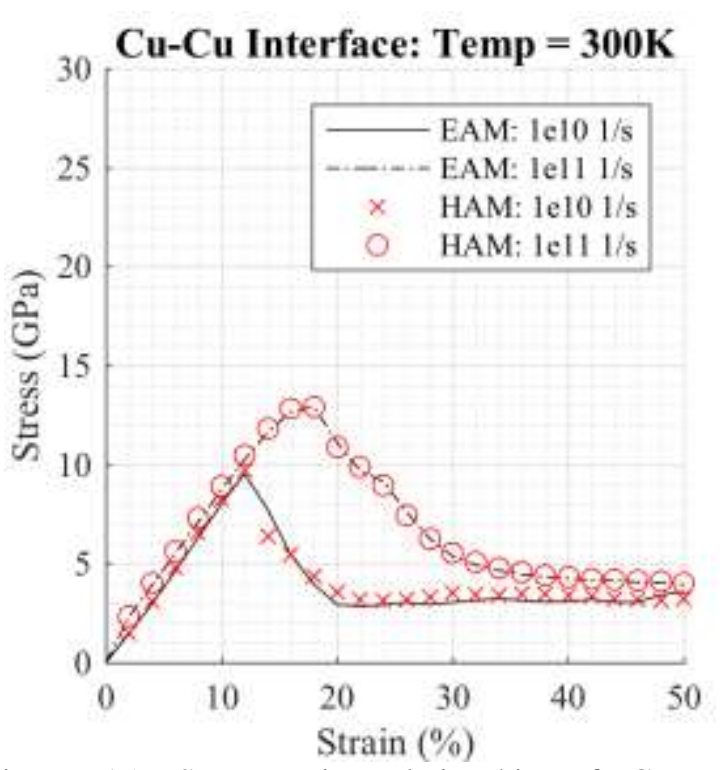

Figure 15: Stress-strain relationship of $\mathrm{Cu}$ EAM compared to HAM at $300 \mathrm{~K}$ temperature and strain rates $10^{10}$ and $10^{11} 1 / \mathrm{s}$.. 


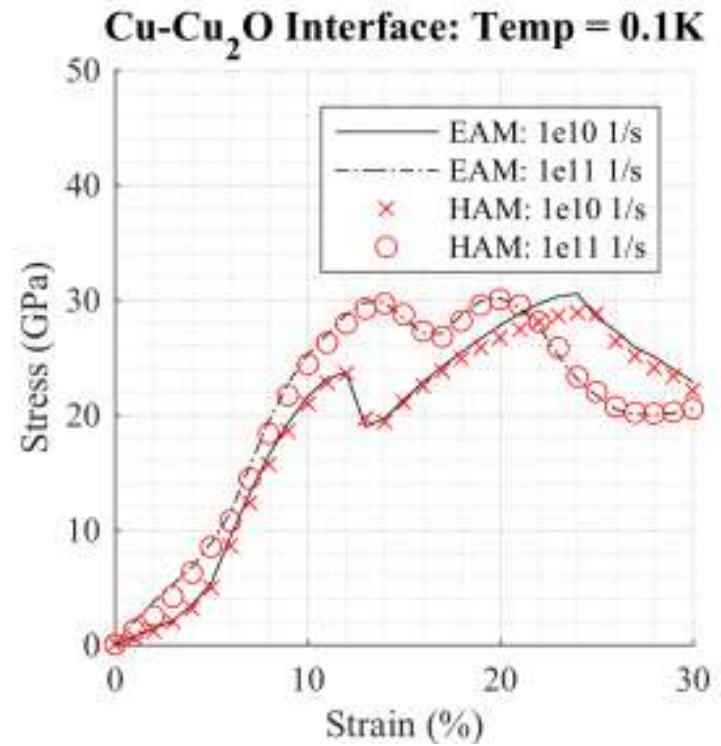

Figure 16: Stress-strain relationship of $\mathrm{Cu}_{2} \mathrm{O}$ control model, COMB, compared to HAM at $0.1 \mathrm{~K}$ temperature and strain rates $10^{10}$ and $10^{11} 1 / \mathrm{s}$

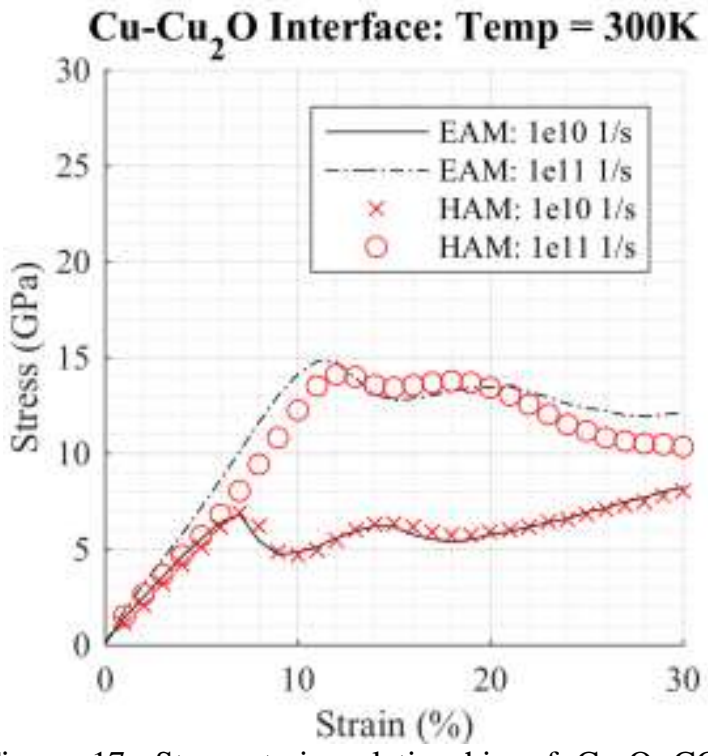

Figure 17: Stress-strain relationship of $\mathrm{Cu}_{2} \mathrm{O}$ COMB compared to HAM at $300 \mathrm{~K}$ temperature and strain rates $10^{10}$ and $10^{11} 1 / \mathrm{s}$.

Finally, to demonstrate the effectiveness of the hybrid adhesion method in a full delamination failure mode, a two-layer structure of aluminum and copper is presented. In the (001) plane, both materials are FCC structures but have dissimilar lattice parameters resulting in a misfit dislocation network at the interface and non-uniform adhesion bond lengths. Although the intended use of this method is for large-scale applications, the minimized state of the interface showing misfit dislocations provides a well-defined network when compared with the EAM model, Figure 18.
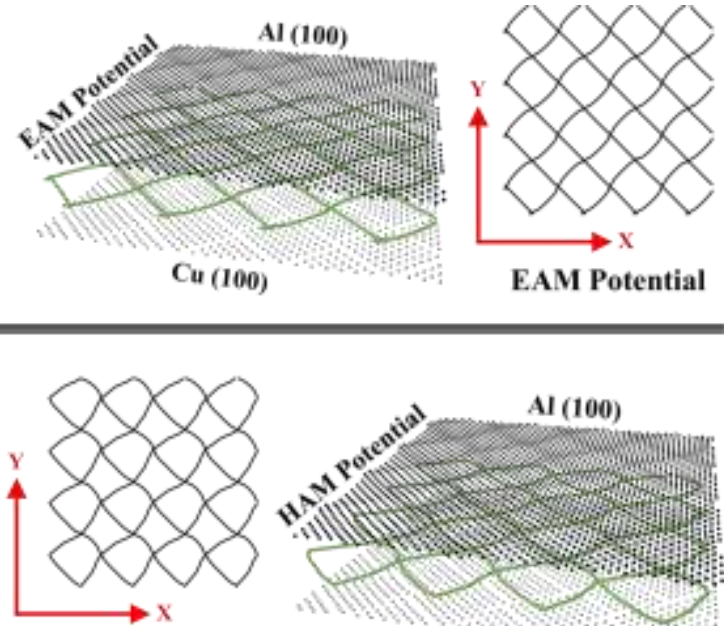

HAM Potential

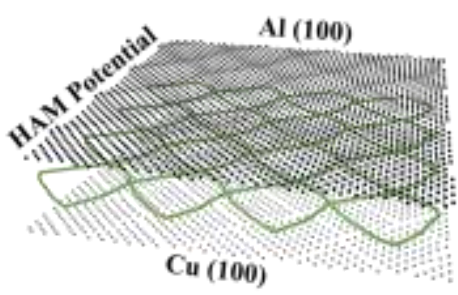

Figure 18: Misfit dislocation network of $\mathrm{Al}-\mathrm{Cu}$ interface using EAM and HAM interatomic potentials.

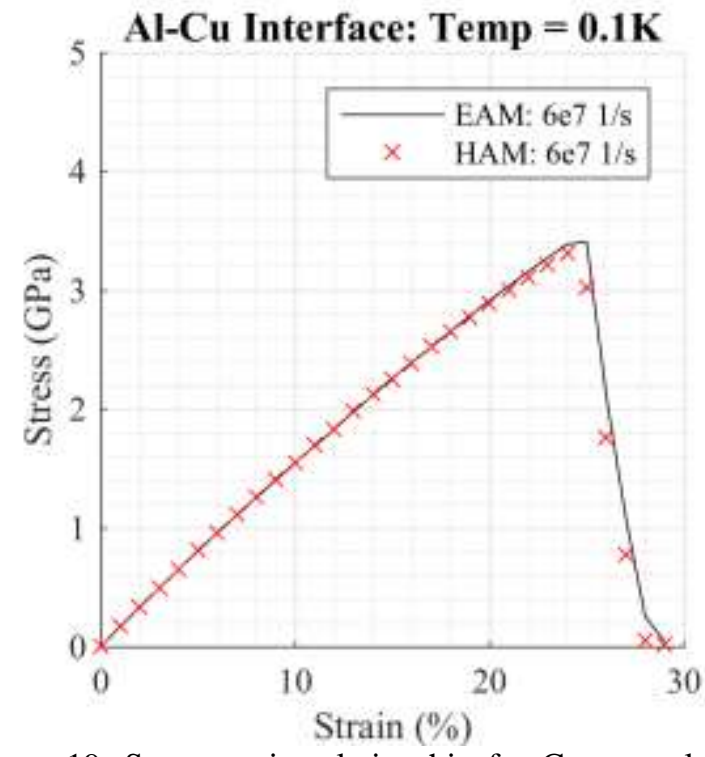

Figure 19: Stress-strain relationship for $\mathrm{Cu}$ control model compared to the HAM model. 
To study the full delamination failure mode, the structure is equilibrated at $0.1 \mathrm{~K}$ for both the EAM control model and the hybrid adhesion method. Unlike the previous full periodic cases, this model is assigned a NVT ensemble with a vacuum space added above the aluminum material layer. To apply a uniaxial load condition to the structure, the bottom two layers of copper lattices are fixed and a velocity in the z-direction is applied the top two rows of aluminum lattices to apply the load. A strain-rate of $6.0 \mathrm{e} 71 / \mathrm{s}$ is maintained until the ultimate yield point is reached for both EAM and HAM models, Figure 19. As with the copper and copper-oxide examples, the hybrid adhesion method captures the bulk mechanical behavior of the structure, including the ultimate yield strength. The hybrid adhesion method also accurately predicts failure in the surface phase region on the copper layer as expected from the EAM simulation, Figure 20.

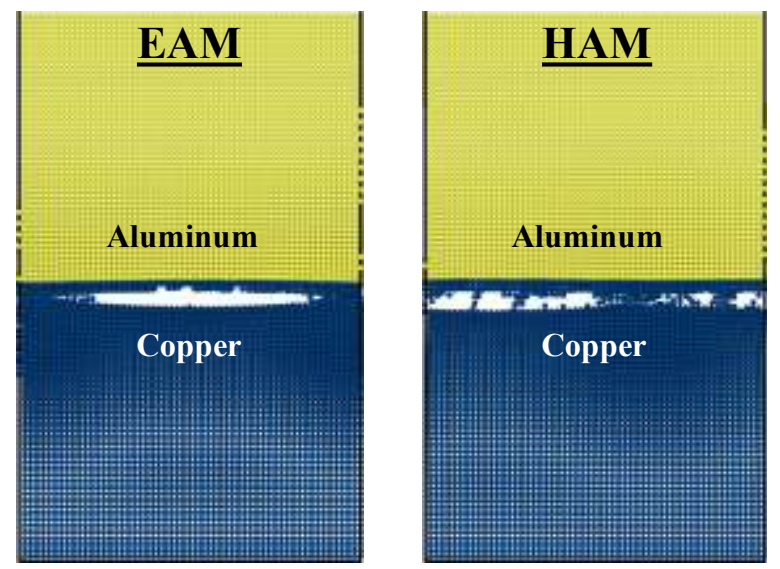

Figure 20: Delamination of $\mathrm{Al}-\mathrm{Cu}$ interface, EAM compared to HAM.

\section{Conclusions}

A new MD hybrid potential modeling approach is developed for the intended use of simulating large-scale mechanical characteristics as well as the delamination phenomena in thin film applications. The hybrid adhesion method was designed to discretize the diffused energy contributions of a material interface into two separate bulk quantities and an adhesive region joining the two layers. This allows for the use of otherwise independent interatomic potentials in the modeling of composite layer interfaces. The methodology identifies a surface adhesion relationship using small-scale first-principle calculations and applies the relationship to an atomistic interface using a distributed array of bonds added at the surface to surface interactions. After calibrating and verifying the applied bond parameters used to define the adhesive strength, the hybrid adhesion method models have been shown to accurately simulate the elastic mechanical properties when compared to traditional modeling techniques. In addition, the hybrid adhesion method models have been verified to show good agreement in the stress-strain relationships of standard tensile load case at various temperatures. The hybrid adhesion method approach presents an alternative to the full development of an interatomic force field when studying new material interactions by limiting the many-body interactions to a predefined surface phase region. Consequently, this results in reduced model preparation and simulation time when studying new material interfaces that do not have fully developed interatomic potentials. 
As an approximation method for modeling the adhesive behavior of complex interfaces, the hybrid adhesion method is limited in application to large scale mechanical behaviors and may not provide the most accurate interface dislocation representations. The nature of the method smears long-range atomic interactions to an array of one-dimensional adhesion bonds used to replicate the average large-scale surface behavior under tensile loading. As a result, this approximation may result in artificial stresses and energies in the surface phase region which could impact the formation and propagation of dislocations in less than ideal interfaces. Therefore, this method may not be appropriate for the study of dislocation nucleation that develop at a material interface under certain loading conditions. While we have shown that this method can accurately reproduce misfit dislocations under equilibrium, the documented work has thus far been limited to atomically flat and ideal cubic interfaces where failure modes are closely controlled. However, for the intended use in composite thin film metallic structures, the hybrid adhesion method has been shown to efficiently and accurately model the mechanical behavior and delamination failure mode at the atomic scale. Additional studies are necessary to determine the full capabilities of the hybrid adhesion method on complex and amorphous material interfaces.

The intended future applications of the hybrid adhesion method approach are geared towards the study of adhesive interactions in thin layer delamination occurrences in consumer electronics. This is an interesting subject due to the wide range of metals, metal-oxides and polymer epoxies composite combinations and the exposure to constant environmental changes, such as temperature fluctuations and moisture content $[8,10,30]$. These factors lead to a failure mode in material delamination where experimental data is plentiful, but computational approximations are currently lacking.

\section{Acknowledgements}

The authors acknowledge the support of the US Department of Energy (DOE) through the AAPP Program for this work. This research used resources provided by the LANL Institutional Computing Program. LANL, an affirmative action/equal opportunity employer, is operated by Los Alamos National Security, LLC, for the National Nuclear Security Administration of the U.S. DOE under contract DE-AC52-06NA25396.

\section{Appendix}

The main purpose of the hybrid adhesion method is to model material interfaces with unique force-fields. The results provided in the main sections of this manuscript demonstrate the application of this approach using the same force-fields for simplicity and to limit the deviations in mechanical response to the method alone. To show the versatility of this approach, the tensile loading condition of the copper interface model at $300 \mathrm{~K}$ has been repeated using the hybrid adhesion method with an EAM-COMB interface definition, Figure 21. The results show comparable mechanic behaviors to the EAM control model, although there may be additional deviations from Figure 15 due to the differences between the COMB and EAM definitions of copper at this temperature. 


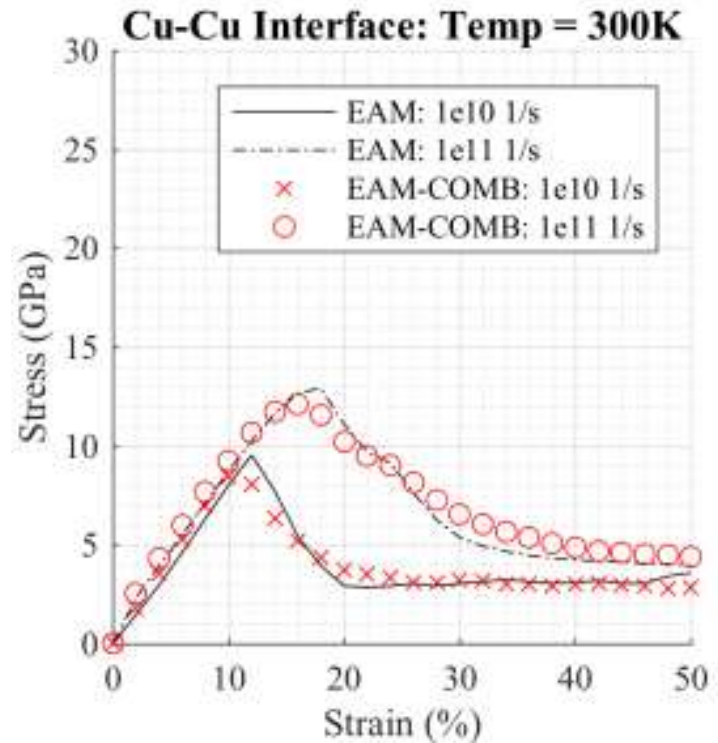

Figure 21: Stress-strain relationship of $\mathrm{Cu}$ interface model, EAM, compared to HAM (EAM/COMB) at $300 \mathrm{~K}$ temperature and strain rates $10^{10}$ and $10^{11} 1 / \mathrm{s}$.

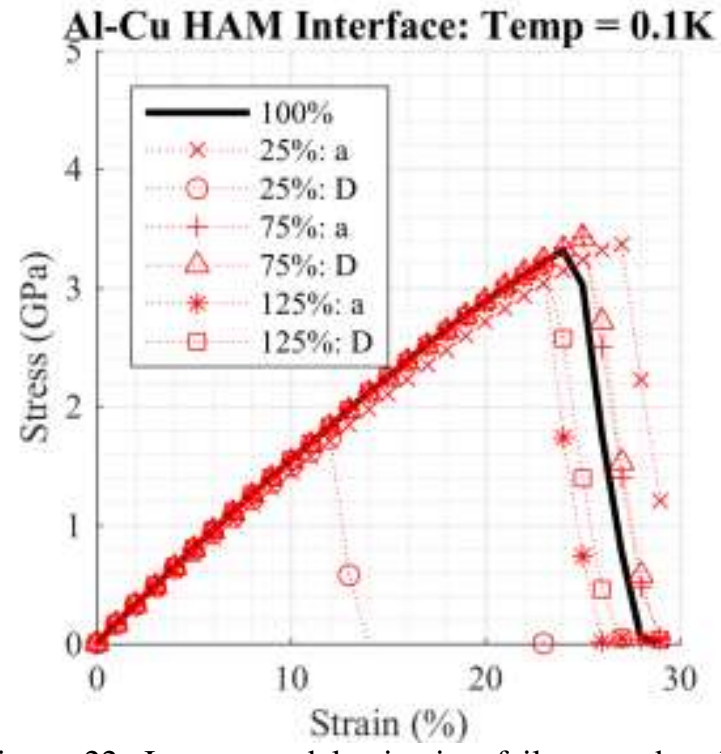

Figure 22: Impact to delamination failure mode when adhesion bond parameters are modified.

In addition, to show the importance of an accurate adhesion bond strength definition on the delamination failure mode, a sensitivity analysis was performed on the strength and shape ( $D$ and a) parameters used to define the adhesion bonds in the copper-aluminum interface model, Figure 22. The two parameters were changed to $25 \%, 75 \%$ and $125 \%$ of the ideal values and rerun for delamination with special focus on the ultimate failure point. The results show that while the elastic properties of the material are largely unchanged, the yield point changes noticeably depending on the parameters.

\section{References}

[1] A.K. Rappé, C.J. Casewit, K. Colwell, W. Goddard Iii, W. Skiff, Journal of the American Chemical Society, 114 (1992) 10024-10035.

[2] M.S. Daw, S.M. Foiles, M.I. Baskes, Materials Science Reports, 9 (1993) 251-310.

[3] M. Mendelev, M. Kramer, R. Ott, D. Sordelet, D. Yagodin, P. Popel, Philosophical Magazine, 89 (2009) 967-987.

[4] J.-M. Zhang, F. Ma, K.-W. Xu, Applied Surface Science, 229 (2004) 34-42.

[5] B. Devine, T.-R. Shan, Y.-T. Cheng, A.J. McGaughey, M. Lee, S.R. Phillpot, S.B. Sinnott, Physical Review B, 84 (2011) 125308.

[6] T.-R. Shan, B.D. Devine, T.W. Kemper, S.B. Sinnott, S.R. Phillpot, Physical Review B, 81 (2010) 125328.

[7] M. Baskes, Materials Chemistry and Physics, 50 (1997) 152-158.

[8] S. Yang, F. Gao, J. Qu, Polymer, 54 (2013) 5064-5074.

[9] A. Hallil, J.-M. Raulot, M. Cherkaoui, Computational Materials Science, 81 (2014) 366-373.

[10] S. Yang, F. Gao, J. Qu, Electronic Components and Technology Conference (ECTC), 2010 Proceedings 60th, IEEE, 2010, pp. 128-134.

[11] R. Dingreville, J. Qu, Journal of the Mechanics and Physics of Solids, 56 (2008) 1944-1954. [12] R. Dingreville, J.M. Qu, M. Cherkaoui, Journal of the Mechanics and Physics of Solids, 53 (2005) 1827-1854. 
[13] R. Dingreville, J. Qu, Computational Materials Science, 46 (2009) 83-91.

[14] H.-J. Butt, K. Graf, M. Kappl, Physics and chemistry of interfaces, John Wiley \& Sons, 2006.

[15] E.D. Shchukin, A.V. Pertsov, E.A. Amelina, A.S. Zelenev, Colloid and surface chemistry, Elsevier, 2001.

[16] M.I. Mendelev, H. Zhang, D.J. Srolovitz, Journal of materials research, 20 (2005) 11461153.

[17] S. Ratanaphan, D.L. Olmsted, V.V. Bulatov, E.A. Holm, A.D. Rollett, G.S. Rohrer, Acta Materialia, 88 (2015) 346-354.

[18] A. Wicaksono, C. Sinclair, M. Militzer, Computational Materials Science, 117 (2016) 397405.

[19] H. Zhang, M. Upmanyu, D. Srolovitz, Acta materialia, 53 (2005) 79-86.

[20] T. Semoto, Y. Tsuji, K. Yoshizawa, The Journal of Physical Chemistry C, 115 (2011) 11701-11708.

[21] R. Posner, O. Ozcan, G. Grundmeier, Water and ions at polymer/metal interfaces, Design of Adhesive Joints Under Humid Conditions, Springer, 2013, pp. 21-52.

[22] T. Semoto, Y. Tsuji, K. Yoshizawa, Bulletin of the Chemical Society of Japan, 85 (2012) 672-678.

[23] S. Plimpton, Journal of computational physics, 117 (1995) 1-19.

[24] A. Arya, E.A. Carter, The Journal of chemical physics, 118 (2003) 8982-8996.

[25] B. Sachs, T. Wehling, M. Katsnelson, A. Lichtenstein, Physical Review B, 84 (2011) 195414.

[26] D. Jiang, E.A. Carter, Physical Review B, 72 (2005) 165410.

[27] Y. Mishin, M. Mehl, D. Papaconstantopoulos, A. Voter, J. Kress, Physical Review B, 63 (2001) 224106.

[28] L.A. Girifalco, V.G. Weizer, Physical Review, 114 (1959) 687.

[29] S. Foiles, M. Baskes, M.S. Daw, Physical Review B, 33 (1986) 7983.

[30] J. Qu, Adhesion and Failure of Polymer-Metal Interfaces in Microelectronic Packaging, Handbook of Damage Mechanics, Springer, 2015, pp. 645-679. 


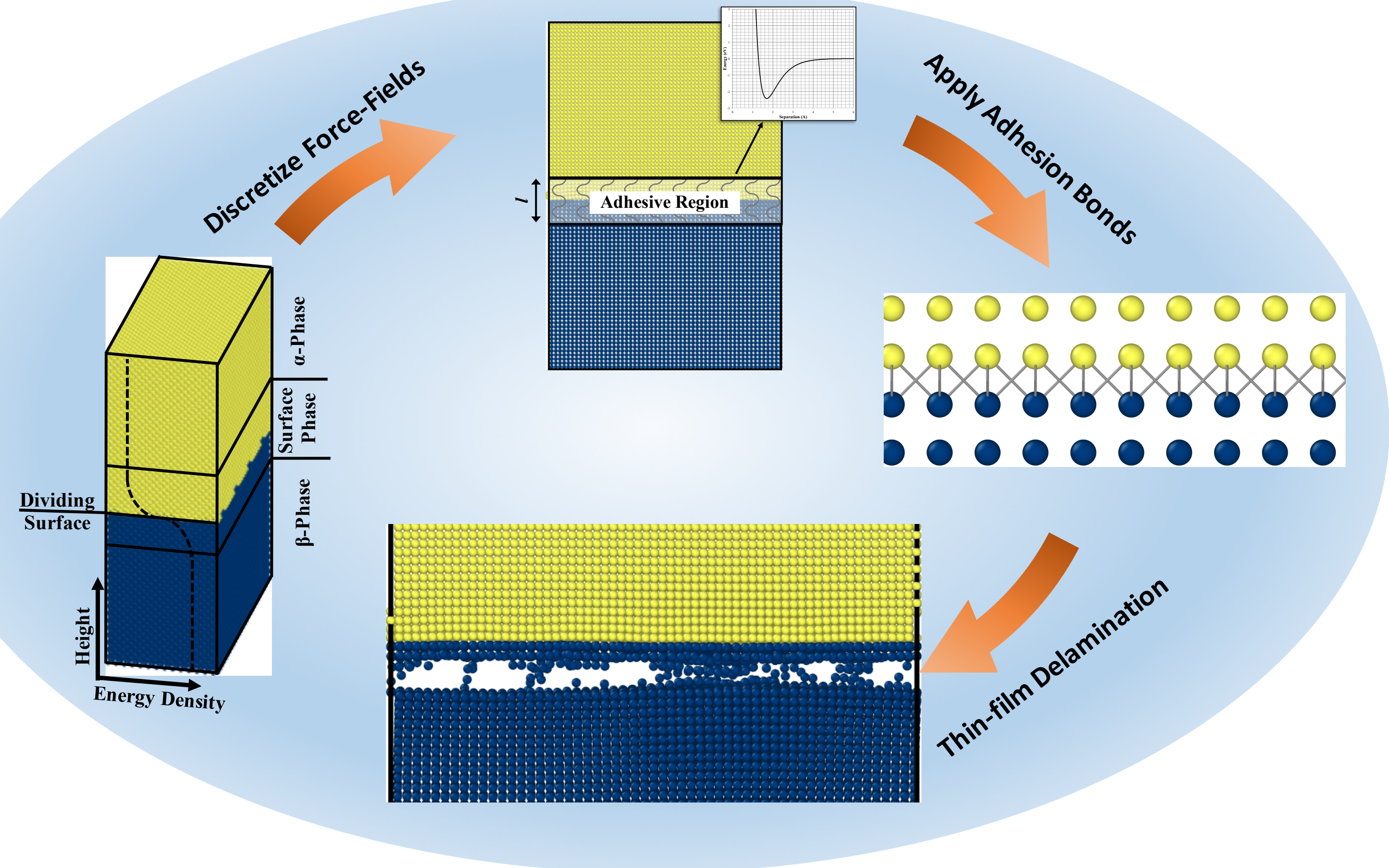

